\title{
Spectroscopic binary mass determination using relativity
}

\author{
Shay Zucker ${ }^{1}$ and Tal Alexander ${ }^{2}$ \\ ${ }^{1}$ Dept. of Geophysics \& Planetary Sciences, Raymond and Beverly Sackler Faculty of Exact \\ Sciences, Tel Aviv University, Tel Aviv 69978, Israel \\ email: shayz@post.tau.ac.il \\ ${ }^{2}$ Faculty of Physics, Weizmann Institute of Science PO Box 26, Rehovot 76100, Israel \\ email: Tal.Alexander@weizmann.ac.il
}

\begin{abstract}
High-precision radial-velocity techniques, which enabled the detection of extra-solar planets, are now sensitive to the lowest-order relativistic effects in the data of spectroscopic binary stars (SBs). We show how these effects can be used to derive the absolute masses of the components of eclipsing single-lined SBs and double-lined SBs from Doppler measurements alone. High-precision stellar spectroscopy can thus substantially increase the number of measured stellar masses, thereby improving the mass-radius and mass-luminosity calibrations.
\end{abstract}

Keywords. binaries: close, binaries: spectroscopic, celestial mechanics, methods: data analysis, relativity, techniques: radial velocities

\section{Introduction}

During the current IAU Symposium, optical Doppler measurements of binary stars were hardly mentioned. Usually, one doesn't expect to see any relativistic effects in the optical Doppler data, apart from long term (secular) effects, with an accumulating nature, such as the relativistic precession of the periastron. However, the recent two decades have witnessed the emergence of precise radial-velocity (RV) measurements, with long-term precisions of a few meters per second, that are now routinely obtained by several telescopes around the world. The most notable scientific achievement of precise RV measurements has been the detection of planets orbiting solar-type stars (Mayor \& Queloz 1995, Marcy \& Butler 1996). In this paper we show that these new techniques are already sensitive to the lowest-order (Einstein delay) terms, and that they can be used to add more data about the binary star, which is not attainable in any other way. Kopeikin \& Ozernoy (1999) have already detailed the relativistic effects one expects to find in the Doppler measurements of binary stars. Here we focus on the effects that we expect to practically measure in SBs, and identify the additional information they may provide. We have already introduced our main arguments in an ApJ Letter (Zucker \& Alexander 2007).

The typical velocities of components of close binary stars can be as high as $150 \mathrm{~km} \mathrm{~s}^{-1}$, $\beta \equiv v / c \sim \mathcal{O}\left(10^{-4}\right)$. The classical Doppler shift formula predicts a relative wavelength shift $\Delta \lambda / \lambda$ of order $\beta$. The next order corrections are $\beta^{2} \sim \mathcal{O}\left(10^{-8}\right)$, which translate to $\mathcal{O}\left(1 \mathrm{~m} \mathrm{~s}^{-1}\right)$. Terms of order $\beta^{3}$ are beyond foreseen technical capabilities. We thus limit our analysis to $\mathcal{O}\left(\beta^{2}\right)$ effects - the transverse Doppler shift (time dilation) and the gravitational redshift. We focus on effects we can detect during relatively short observing runs, so we ignore the higher-order secular terms. 


\section{Single-lined spectroscopic binary}

The Keplerian RV curve of a single-lined spectroscopic binary (SB1) can be presented as:

$$
V_{R 1}=K_{1} \cos \omega \cos \nu-K_{1} \sin \omega \sin \nu+e K_{1} \cos \omega+V_{R 0},
$$

where $K_{1}$ is the primary star RV semi-amplitude, $\omega$ is the argument of periastron, $e$ is the eccentricity, $V_{R 0}$ is the center-of-mass RV, and $\nu$ is the time-dependent true anomaly. The customary procedure to solve an SB1 is to fit this orbital model to the observed RV data. This fit is achieved through some optimization algorithm that scans the $(P, T, e)$ space (period, periastron time and eccentricity). For each trial set of values for these three parameters, the algorithm produces the corresponding $\nu(t)$ and then solves analytically for $K_{C 1}\left(=K_{1} \cos \omega\right), K_{S 1}\left(=-K_{1} \sin \omega\right)$, and $V_{R 0}$, which appear linearly in the expression for $V_{R 1}$.

We have recently shown (Zucker \& Alexander 2007) that including the $\beta^{2}$ effects changes Equation 2.1 by simply modifying the linear elements $K_{S 1}, K_{C 1}$ and $V_{R 0}$. The modified elements are given by:

$$
\begin{aligned}
& K_{S 1}^{\prime}=-K_{1}\left(1+\frac{V_{R 0}}{c}\right) \sin \omega \\
& K_{C 1}^{\prime}=K_{1}\left[\left(1+\frac{V_{R 0}}{c}\right) \cos \omega+\frac{e}{\sin ^{2} i} \frac{2 K_{1}+K_{2}}{c}\right] \\
& V_{R 0}^{\prime}=V_{R 0}+\frac{1-e^{2}}{\sin ^{2} i} \frac{K_{1}}{c}\left(\frac{3}{2} K_{1}+K_{2}\right)+\frac{V_{0}^{2}}{2 c} .
\end{aligned}
$$

The modified linear elements are more difficult to interpret now. The three quantities $K_{S 1}^{\prime}, K_{C 1}^{\prime}$, and $V_{R 0}^{\prime}$ depend on the six elements $K_{1}, K_{2}, \omega, \sin i, V_{R 0}$, and $V_{0}$. Thus, Equations (2.2) are under-determined and we cannot completely infer the six elements above, unless some additional independent information is available, or further assumptions are introduced.

Such independent information may be available through precise photometry of eccentric eclipsing binaries. There, the shapes and widths of the eclipses as well as the phase differences between primary and secondary eclipses can be used to estimate $\omega$ and $\sin i$. In this case, we may derive $K_{2}$ - the RV amplitude of the secondary star. By obtaining $K_{2}$ we effectively turn the binary into a double-lined spectroscopic binary (SB2), in which both $K_{1}$ and $K_{2}$ are measured. Together with the known inclination, we then obtain full knowledge of the component masses.

Curiously, another result of including the relativistic terms is that an eccentric binary should always display an apparent RV signature, even in the extreme case where the inclination is exactly zero and the orbit is observed face on. Nevertheless, such small values of the inclination are extremely rare and this possibility is not realistic.

\section{Double-lined spectroscopic binary}

In the case of a Keplerian SB2, there are two sets of measured RVs. The two sets of RVs share the same fundamental orbital elements $\left(P, T, e, \omega\right.$, and $\left.V_{R 0}\right)$, and the only difference between them is their amplitudes $K_{1}$ and $-K_{2}$. The common procedure is to scan the space of the four parameters $(P, T, e, \omega)$, and then solve analytically for the three linear elements $K_{1}, K_{2}$, and $V_{R 0}$. However, when we incorporate the relativistic corrections above, we see that we now have two RV curves with two different sets of 
derived amplitudes, arguments of periastra, and center-of-mass velocities. The two RV curves still share the same period, periastron time and eccentricity.

The four equations corresponding to the equations $2.2 a$ and $2.2 b$ have four unknowns: $K_{1}, K_{2}, \omega$, and $\sin i$. $V_{R 0}$ appears in these four equations always divided by the speed of light. Thus, we can safely use its approximate derived value, from either set of measured velocities, since the discrepancy will be only $\mathcal{O}\left(\beta^{3}\right)$. Solution of this set of equations will yield a more accurate estimate of the first three unknowns, but more importantly, it will yield an estimate of $\sin i$. Retaining only leading order terms, we can arrive at the following solution:

$$
\sin ^{2} i=\frac{3 e}{\omega_{2}^{\prime}-\omega_{1}^{\prime}} \frac{K_{S 2}^{\prime}+K_{S 1}^{\prime}}{c},
$$

where $\omega_{1}^{\prime}$ and $\omega_{2}^{\prime}$ are the apparent arguments of periastra of the two components. The fact that the two arguments of periastra differ is a pure relativistic effect. Note that classically, there is no way to estimate $\sin i$ from pure RV data alone. The value of $\sin i$ is usually obtained only from the analysis of SBs that are eclipsing or astrometric binaries.

\section{Discussion}

We present here an approach to extract more information from RV data of a spectroscopic binary, based on the inclusion of relativistic effects. The practical potential lies in the time-dependent parts of the relativistic terms which are closely linked to the variation of the distance between the binary components. Thus, these terms are especially useful for orbits that are eccentric enough.

The approach is mainly useful in the case of SB2s, but precise photometry or astrometry may add the required information to utilize relativity in SB1s as well. Precise photometry space missions, like MOST (Walker et al. 2003), CoRoT (Baglin et al. 2003), and Kepler (Basri et al. 2005), may be able to provide precise enough measurements of $\omega$ and the inclination of eclipsing binaries from the analysis of their light curves.

In any case, it is essential that the data quality be high enough to be sensitive to variations of the required order, namely one meter per second or less. Currently, the best precision is obtained by the ESO HARPS fiber-fed echelle spectrograph, where the RV error can be as low as $1 \mathrm{~ms}^{-1}$ in certain cases and maybe even less (Lovis et al. 2005). In the future, much better precisions can be hoped for, on instruments designed for the "extremely large telescopes" (e.g., Pasquini et al. 2006).

Currently, precisions of a few meters per second are still difficult to obtain, and besides using the best instruments available, there are also several limitations imposed by the star itself. Thus, early-type stars or rapid rotators, where the spectral lines are significantly broadened, do not lend themselves easily to high-precision RV measurements. Stellar oscillations and star spots are also a concern as they can cause apparent RV modulation. In addition, analyzing SB2s with the same level of precision as SB1s has not been easy until recently, when TODCOR (Zucker \& Mazeh 1994) was applied successfully to highprecision spectra by several teams (Zucker et al. 2004,Udry et al. 2004,Konacki 2005).

The effects we have examined are obviously most useful when the orbits are eccentric and the RV amplitudes are large enough. Large RV amplitudes are typical for close binaries, which are expected to have undergone orbital circularization and usually have vanishing eccentricities. However, eccentricity somewhat increases the RV amplitude, and even relatively wide binaries, with high enough eccentricities, can display quite large RV amplitudes, as the next two paragraphs demonstrate. 


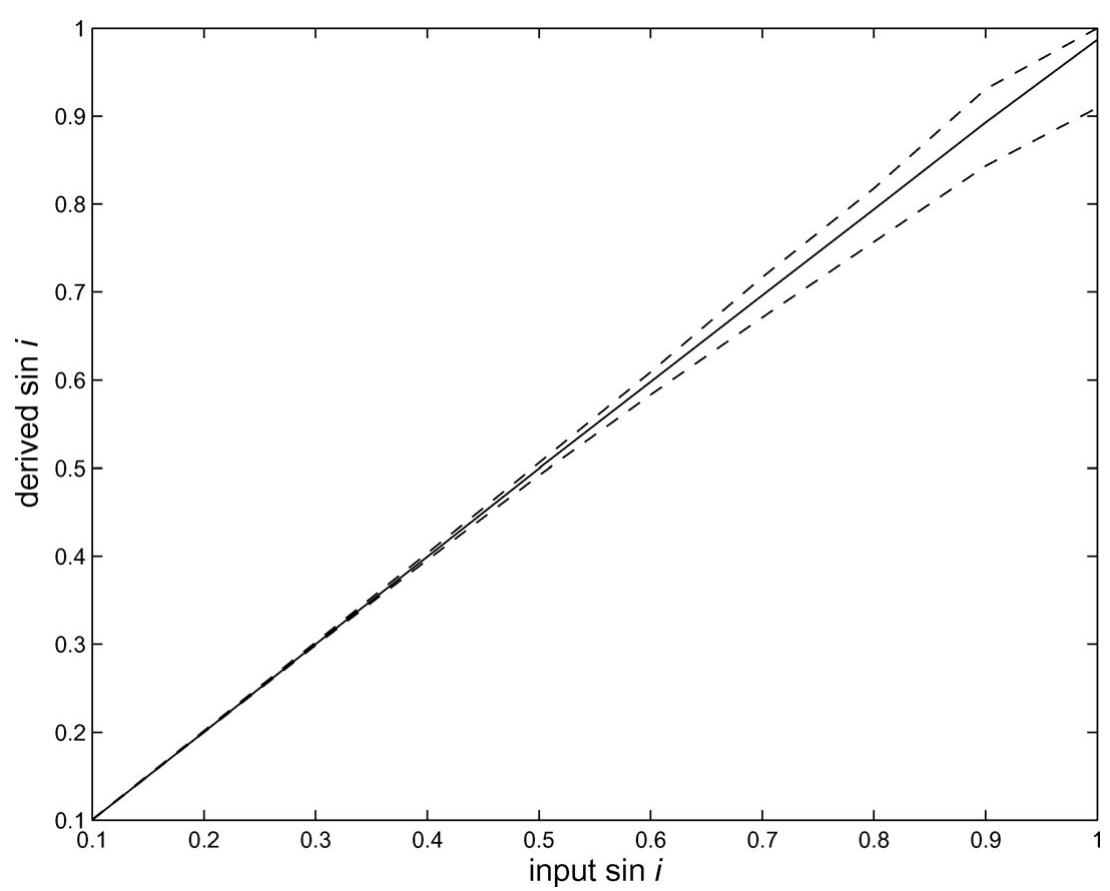

Figure 1. The results of simulations of 12 Boo including relativistic effects, assuming RV precision of $1 \mathrm{~m} \mathrm{~s}^{-1}$. The plot shows percentiles of the derived $\sin i$ against the input simulated value. The dashed lines represent the first and third quartile, and the solid line is the median.

To demonstrate the relevance of the suggested approach in real-life cases we chose to examine the SB2 12 Boo. Recently, Tomkin \& Fekel (2006) published a precise solution of 12 Boo based on RVs obtained at the 2.1-m telescope at the McDonald Observatory and at the Coudé feed telescope at Kitt Peak National Observatory, with RV precisions of $0.1-0.2 \mathrm{~km} \mathrm{~s}^{-1}$. The system has a period of 9.6 days, eccentricity 0.2 , and both RV semiamplitudes are close to $70 \mathrm{~km} \mathrm{~s}^{-1}$. These orbital parameters translate to an expected relativistic amplitude variation of about $10 \mathrm{~m} \mathrm{~s}^{-1}$. Furthermore, with a declination of $+25^{\circ}$, the star is observable by HARPS. Its brightness (5th magnitude) and spectral type (F9IV) make it fairly reasonable to expect a precision of $1 \mathrm{~m} \mathrm{~s}^{-1}$ with HARPS. We used the available 24 RVs from Tomkin \& Fekel (2006) and augmented them with only 3 simulated HARPS measurements (assuming errors of $1 \mathrm{~m} \mathrm{~s}^{-1}$ ), including the relativistic effects. For each assumed value of $\sin i$ we produced 1000 sets of simulated measurements and solved for the orbital elements, using Equation 3.1 to estimate $\sin i$. Figure 1 shows the median of the derived values in solid line, and the $25 \%$ and $75 \%$ percentiles in dashed lines.

The figure demonstrates that with reasonable efforts, $\sin i$ can be measured satisfactorily. In the worst case where $\sin i=1$, the standard deviation of the derived inclination is 0.14 and a few more precise measurements can reduce this value significantly. An additional advantage of this test case is that the inclination of 12 Boo has already been measured by interferometry and is known to be $108^{\circ}$ (Boden et al. 2005). In real observations, more than three precise measurements may be needed in order to account for differences in zero points between instruments. In fact we have already secured several HARPS exposures of 12 Boo and after some technical difficulties, we are now at the process of analysing it.

Care must be taken to model correctly any other effects of order $\beta^{2}$ that might contaminate the data. One such effect is the light-travel-time effect. This effect can be easily 
approximated to the relevant order by adding the following term to $V_{R 1}$ (and a corresponding one to $\left.V_{R 2}\right)$ :

$$
\Delta V_{\mathrm{LT}}=\frac{K_{1}^{2}}{c} \sin ^{2}(\nu+\omega)(1+e \cos \nu) .
$$

Another effect which should be analyzed carefully is the tidal distortion of the stellar components, in particular close to periastron. This distortion may affect the spectral lines, introducing line asymmetry, which can bias the estimated Doppler shift. RV extrasolar planet surveys use the line-bisector analysis (e.g., Queloz et al. 2001) to quantify such time-dependent asymmetries. Further development of this technique may be the key to disentangle the tidal distortion and the relativistic effects.

One important application of the proposed method is to calibrate the low-mass end of the mass-luminosity relation, to better understand the stellar-substellar borderline. This mass regime is still poorly constrained, since low mass SB2s are quite rare due to the special photometric, spectroscopic and geometric requirements (Ribas 2006). Large efforts are in progress to obtain accurate stellar masses in this regime, including adaptive optics, interferometry and in the future space interferometry (e.g., Henry et al. 2005). We propose a new, relatively accessible tool to accomplish this goal, where the only requirements are spectroscopic. Precise RVs for low-mass SB2s were already measured by Delfosse et al. (1999). Using the method presented here, their absolute masses may be derived with a relatively small observational effort. No other method exists yet to derive this information purely from RV measurements.

\section{Acknowledgment}

S.Z. wishes to express his gratitude to the organizing committees for their support in the form of a travel grant.

\section{References}

Baglin, A. 2003, Adv. Sp. Res., 31, 345

Basri, G., Borucki, W. J., \& Koch, D. 2005, New Astron. Revs, 49, 478

Boden, A. F., Torres, G., \& Hummel, C. A. 2005, ApJ, 627, 464

Delfosse, X., Forveille, T., Beuzit, J.-L., Udry, S., Mayor, M., \& Perrier, C. 1999, A\& A, 344, 897

Henry, T. J., et al. 2005, BAAS, 37, 1356

Konacki, M. 2005, ApJ, 626, 431

Kopeikin, S. M. \& Ozernoy, L. M. 1999, ApJ, 523, 771

Lovis, C., et al. 2005, A\&A, 437, 1121

Marcy, G. W. \& Butler, R. P. 1996, ApJ, 464, L147

Mayor, M. \& Queloz, D. 1995, Nature, 378, 355

Pasquini, L., et al. 2006, in IAU Symp. 232, The Scientific Requirements for Extremely Large Telescopes, ed. P.A. Whitelock, M. Dennefeld \& B. Leibundgut (Cambridge: Cambridge Univ. Press), p. 193

Queloz, D., et al. 2001, A\&A, 379, 279

Ribas, I. 2006, ApESSS, 304, 89

Tomkin, J. \& Fekel, F. C. 2006, AJ, 131, 2652

Udry, S., Eggenberger, A., Mayor, M., Mazeh, T., \& Zucker, S. 2004, Rev. Mexicana AyA, 21, 207

Walker, G., et al. 2003, PASP, 115, 1023

Zucker, S. \& Alexander, T. 2007, ApJ, 645, L83

Zucker, S. \& Mazeh, T. 1994, ApJ, 420, 806

Zucker, S., Mazeh, T., Santos, N. C., Udry, S., \& Mayor, M. 2004, A\&\&A, 426, 695 